\title{
ATTD 2018 Abstract Author Index
}

Abdi, Z., 362

Abdullah, N.R.A., 356

Abitbol, A., 44

Abraham, M.B., 205

Abreu, A., 329, 330

Acciaroli, G., 45, 133, 203

Acerini, C.L., 75, 128

Adachi, D.C., 343

Adamikova, A., 142

Addaguduru, S., 86

Adolfsson, P., 209

Advani, A., 227

Afshari, M., 249

Aghaei Meybodi, H.R., 249

Agrawal, P., 38, 211, 234

Aguayo, G.A., 76, 125

Aiello, V., 169

Ajjan, R., 51

Akhter, S., 334

Al Hayek, A., 364

Al Zyoud, M., 264

Al-Alosi, A., 99

Alarcon, J., 317

Alcantara Aragon, V., 333

Aldungarov, E., 348

Alexeeva, R., 102

ALfayez, F., 352

Ali Khalaf, F., 264, 289, 358

Alina, A., 348

Alipour, M., 362

Alipour, R., 362

Aliste, M., 275

Allen, J., 75, 128

Alluis, B., 304

Altendorfer-Kroath, T., 313

Alva Karinka, S., 198

Alvarez, H., 337

Åm, M.K., 105

Amato, A.M.L., 203

Amiel, S., 232

Amritha, M.K., 335

Amzaleg, M., 158

Anantharaja, S., 52

Anastassiadis, E., 304

Andersen, G., 64

Anderson, S., 106

Andre, A., 327

Andrieu, J.M., 136, 137

Anilkumar, A.L., 115

Anthimopoulos, M., 258

Antillon Ferreira, C.A., 92

Arampatzis, S., 258
Arens, J.H., 84

Ariel, T., 165

Arnaldi, C., 230

Arnolds, S., 123, 130

Aronson, R., 44

Arroyo-Díez, F.J., 46

Årsand, E., 90, 154, 240, 244, 248, 251, $339,353,354$

Arteiro, C., 286

Arunachalam, S., 211

Assy, M., 32

Astudillo, R., 275

Atakov-Castillo, A., 173, 174

Atay, Z., 280

Atlas, E., 24, 42, 165

Augustin, T., 284

Avari, P., 29, 148

Avivit, B., 165

Avogaro, A., 203

Ayaz, T., 366

Azkutia, A., 212

Babu, V., 310

Baena-Nieto, M., 220

Baggott, A., 294

Bahartan, K., 199

Bailey, T., 39, 40, 94

Bailey, T.S., 312

Baillot-Rudoni, S., 200

Bain, S., 63

Bajaj, S., 36, 170

Balcazar, C., 329

Balcazar, C.M., 330

Balliro, C., 28, 124

Bally, L., 123, 130

Balo, A.K., 268

Baltazar, R., 286

Banke, S., 238

Bao, Y., 153

Barana, L., 276

Baraowj, Z., 264

Barbakadze, G., 345

Barber, N., 75, 128

Barnard, K., 113

Barnea, R., 71

Baron, H., 309

Barrasso, M., 219, 254

Barrio-Castellanos, R., 46

Barsukov, I., 269, 270

Basanov, U., 157

Baskaran, V., 241

Bastidas, O., 329
Bastide, N., 134, 136, 137

Basu, A., 68

Basu, R., 68

Battelino, T., 24, 30, 42, 67, 165, 225

Bazdarska, Y., 201

Beato-Vibora, P.I., 46

Beccuti, G., 165

Beck, P., 135

Beck, R., 26

Bee, Y.M., 328

Bellastella, G., 285

Belloso, W., 25

Beltrand, J., 103

Bem, R., 222, 242

Benesch, C., 123, 130

Benitez-Aguirre, P., 98

Benoit, H., 164

Bequette, B.W., 120

Bergenstal, R., 181

Bergman, G., 171

Berria, R., 94

Berry, E., 336

Bertachi, A., 202

Bertaina, S., 276, 277

Bertsch, K., 85

Beruto, V., 25

Besser, R., 128

Beuleke, M., 258

Biagi, L., 202

Bialonczyk, D., 243

Biester, S., 20, 24

Biester, T., 24, 42, 101, 165

Bigi, S., 254

Bird, D., 257, 355

Birngruber, T., 313

Bisio, A., 277

Bitoska, I., 342

Bitovska, I., 287, 288

Bitton, G., 180

Bjornstrom, K., 57

Blauw, H., 107

Blixgård, H., 154

Blomberg, A., 360

Blonde, L., 312

Bocchi, F., 290

Bode, B., 39, 40, 61, 77, 303

Bodwden, C., 299

Bolaños, F., 344

Bolinder, J., 18, 47

Bolla, A.M., 219, 254

Bolli, G.B., 62

Bollow, E., 101 
Bologna, O., 301

Bonadonna, R., 129

Boncompagni, A., 290

Bondia, J., 186, 202

Bonfanti, R., 230

Bonnemaire, M., 69

Bonora, E., 129

Bonura C., 165

Bonura, C., 230

Booth, R., 77

Borda, M.G., 93

Bortz, D.M., 74

Boscari, F., 203

Bösch, P.C., 105, 350

Boselli, M.L., 129

Bosi, E., 156, 254

Bosnyak, Z., 62, 94, 312

Bottari, S.P., 192

Bótyik, B., 331

Bouillet, B., 200

Bowering, K., 65, 303

Boyadzhiev, V., 201

Brackenridge, A., 232

Bradley, S., 306

Bradway, M., 90, 154, 240, 244, 353

Brancato, D., 169

Brandão, P., 260

Brangani, C., 129

Bratina, N., 24, 30, 42, 103, 165, 225

Breton, M., 25, 204

Broglio, F., 277

Brown, D., 281

Brown, D.R., 343

Brown, S., 108

Brož, J., 142, 251

Bruttomesso, D., 203

Bruzzi, P., 165, 290

Buchholtz, K., 65

Buckingham, B., 26, 27, 39, 40, 82, 117, 183

Budiman, E.S., 207

Budin, G., 126

Buffier, P., 200

Builes-Montaño, C., 337

Builes-Montaño, C.E., 344

Burckhardt, M.A., 205, 209

Burnadze, K., 345

Büsing, S., 101

Bustamante Martinez, J.F., 92

Butler, A.T., 206

Cabral, M., 164

Cadieux, I., 273

Caicedo, M.A., 188

Caiulo, S., 165

Calle Fernández, J.R., 212

Calle Pascual, A.L., 212

Calliari, L.E., 12

Calm, R., 175

Calsen, S.M., 53

Cameron, F., 120

Campany, G., 319

Campbell, F., 75, 103

Campos Nañez, E., 25
Campos-Nañez, E., 207, 112

Cano Gutiérrez, C.A., 93

Capelle, D., 200

Cappello, D., 89

Cappon, G., 155

Caprio, M., 58

Caputo, M., 285

Caretto, A., 219, 254

Cargnelutti, K., 30

Carling, J., 281

Carlsen, S.M., 105, 111

Carrano, M., 346, 351

Carvajal, R., 330

Carvalho, D., 260, 286

Casanova, M.E., 329, 330

Casanueva, F., 223

Casas, A., 179

Casciano, O., 285

Castaldo, F., 285

Castaño, O., 330

Catargi, B., 138

Catena, G., 58

Catozzi, D., 277

Ceceña Gonzalez, M.P., 92

Čechová, K., 142, 222

Celebic, A., 76, 125

Cengiz, E., 11, 21, 26

Chan, J.C., 210

Chandarana, H., 55

Chang, Y.Y., 177, 190, 207

Charleer, S., 48

Chatzakis, C., 91

Chaugule, S., 218

Chavarro-Carvajal, D., 93

Chavda, V., 55

Chawla, M., 13, 170

Chawla, P., 13

Chawla, R., 36, 170

Chen Hanna, R., 71

Chen, C.H., 23

Chen, X., 181

Chen, X.O., 44, 86, 208

Cheng, A., 62

Cheng, S.Y., 23

Cherñavvsky, D., 25

Cherubini, V., 103

Chetty, T., 209

Chica, L., 305

Cho, Y.H., 98

Choi, S., 109

Chomutare, T., 354

Choudhary, P., 78, 232, 271

Chow, E., 210

Christe, M., 306

Christensen, S., 238

Christiansen, M., 27, 117

Christiansen, S., 53

Christiansen, S.C., 105, 111

Chrzanowska, J., 81, 302

Chudasama, D., 55

Chung, T.H., 179

Cigler, M., 284

Cinzia, D., 276

Cirillo, P., 285
Clarke, J., 77

Clavel, S., 134, 136, 137

Cobelli, C., 35, 37, 237

Cocca, A., 301

Cohen, N., 75

Cohen, N.C., 128

Cohen, O., 211

Cokolic, M., 357

Colmegna, P., 25

Colon, C.A., 305

Confetto, S., 301

Conget, I., 50, 88, 202, 213

Cordero, T., 38, 39, 40, 181, 182

Costa, A., 286

Coucke, B., 96

Courrèges, J.P., 134, 136, 137

Cox, A., 306

Cozzolino, P., 156

Craig, M., 98

Crevisy, E., 200

Cruzado-Begines, C., 220

Cuder, G., 135

Cuesta, M., 212

Curto, S., 301

D'Angelo, A., 58

D'Angiolella, L.S., 156

d'Annunzio, G., 230

Dachis, J., 261

Daenschel, I., 250, 253

Daenschel, W., 250, 253

Dalla Man, C., 35, 37

Damiano, E., 28, 124, 131

Danne, T., 1, 24, 42, 165

Darenskaya, M., 323, 324, 325

Dario, P., 214

Darssan, D., 257

Dart, J., 41

Dassau, E., 4, 27, 117, 165

Dauchy, A., 69

Dauriz, M., 129

David, A., 80, 115, 340

Davidson, J., 157

Davidson, M.B., 157

Davies, M., 63

Davis, E., 41

Davis, E.A., 205, 209

De Battista, H., 25, 184

De Beaufort, C., 75, 76, 125

de Bock, M., 41, 209

de Campo, A., 135

De la Garza Hernandez, N.E., 92

de Leiva, A., 333

De Melo Dias, C., 76

de Portu, S., 57, 78, 271

De Rosa, P., 346, 351

De Schepper, J., 247

De Vries, H., 2, 5, 9

Dedov, I.I., 283

DeHennis, A., 86, 208

Deja, G., 274

Del Re, L., 89, 166, 229

Delanoë, F., 138

Delbaere, A., 57, 78, 271 
Delgado, E., 69

Dellva, M.A., 66

Dellweg, S., 123, 130

Demina, A., 269, 270

Demircik, F., 151, 152

Demissie, M., 65

Demmitt, J., 226

Derosa, G., 58

Derossi, C., 277

Dethlefsen, C., 310

Devisme, C., 62

Devisscher, C., 247

DeVries, H., 67

DeVries, J.H., 107, 108

Di Martino, R., 346, 351

Di Noto, A., 169

Diaz-Barriga Menchaca, A.P., 92, 245

Diaz, D., 110

Didangelos, T., 95, 272

Diez, J.L., 186

Dimitriadis, G., 69

Dinesen, A., 110

Ding, H., 355

Dirnena-Fusini, I., 105, 111

Dokken, B., 273

Donaghue, K., 98

Donald, R., 231

Dong, H., 255

Douvas, A., 112

Dovc, K., 24, 30, 42, 225

Doyle, F.J., 3

Dozio, N., 219, 254

Dragan, K., 104

Dreval, A., 150, 269, 270

Dubsky, M., 242

Dunger, D.B., 128

Dunn, L.J., 268

Dunn, S., 60

Dunn, T., 33, 47, 49, 51

Dupont, O.N., 348

Dupuy, O., 134, 136, 137

Duracher, D., 126, 321

Ecktein, E., 101

Edelman, S., 63

Edwards, S., 174

Eisler, G., 110

Ekhlaspour, L., 82, 183

Ekong, D., 241

El Darsy, N., 264

El Gamal, M., 264, 289

El-Khatib, F., 28, 124

Elawwa, A., 264, 289, 358

Elena, G., 276

Elena, H.M., 333

Ellingsen, R., 105, 350

Elshowaya, S., 326

Engel, T., 234

English, P., 367

Erez, D., 165

Escobedo Ortiz, A., 245

Espinasse, M., 308, 309

Esposito, K., 285

Esqueira-Sampayo, M.J., 223
Esteves Sanchez, P., 245

Esteves, C., 260, 286

Esteves, J.V., 70

Evans, M., 67, 123

Evans, M.L., 130

Fabian Mauricio, L.V., 214

Fabian, L.V., 246

Fabio, B., 276

Facchinetti, A., 45, 133, 155, 203, 237

Fagher, K., 87

Faradji, R., 92

Faradji, R.N., 245

Farmen, M., 306

Farnan, J., 273

Farrington, C., 128

Fatehi, F., 257, 355

Fejfarová, V., 222, 242

Feng, K., 319

Fernandez-Deus, F.M., 223

Ferrante, R., 169

Ferrara, A., 351

Ferreira Hermosillo, A., 245

Fiala, D., 251

Fichelle, M., 76, 125

Figueroa Andrade, M.H., 245

Filipsson, K., 87

Flacke, F., 63, 177, 314

Fleres, M., 169

Florez, H., 309

Floros, D., 91

Forlenza, G., 26, 27, 39, 40, 117

Forsander, G., 103

Foskett, D., 60

Fougner, A.L., 105, 350

Fourmont, C., 200

França, S., 79

Francesco, Z., 301

Francois-Heude, M., 126

Frandsen, H.A., 69

Franek, E., 61

Freckmann, G., 54, 297, 298, 359

Friedmann, Y., 160

Frisvold, R., 224

Fritsch, M., 101

Fritzen, K., 96

Fröhlich-Reiterer, E., 75

Frongia, A.P., 230

Fukui, T., 149

Fulcher, G., 99

Fulcher, G.R., 293

Funnell, M., 261

Furuya, D.T., 70

Gal, A., 336

Galasso, S., 203

Galindo Rubio, M., 212

Gallo, F., 139

Gamarra, E., 277

Gandolfi, A., 254

Garcetti, R., 226

Garcia Seco, F., 216

Garcia Seco, J.A., 216

García-García-Doncel, L., 220
García-Jaramillo, M., 144

Garcia-Lopez, J.M., 223

Garcia-Tirado, J., 337

Garcia, A., 45

García, M., 246

Garelli, F., 25, 118, 184

Gareth, D., 194

Garg, S., 226

Garibaldi, M., 327

Garza, K., 113

Gaudier, M., 64, 304, 321

Gaudio, G., 58

Gawel, W.B., 274

Gazzaz, Z.J., 97

Georgiou, P., 127, 163, 255, 262

Gerring, D., 315

Ghanassia, E., 134, 136, 137

Gharipour, M., 322

Ghatak, A., 128

Giannoulaki, P., 272

Gicchino, M., 285

Giegerich, C., 37

Gies, I., 247

Gil-Poch, E., 46

Gillard, P., 48

Giménez, M., 148, 186, 46, 50, 88, 202, 213

Gimmon, M., 199

Ginossar, G., 71

Giordanengo, A., 154, 240, 244, 353,354

Girelli, A., 278

Giri, D., 165

Giugliano, D., 285

Glover MBE, D., 160

Gnusina, S., 323, 324, 325

Godoy, J.L., 122

Goh, S.Y., 328

Goik, O., 274

Goldstein, O., 180

Gomez Cruz, J.R., 245

Gómez Medina, A.M., 305

Gómez, A.M., 93, 140, 144, 214, 246

Gonder-Frederick, L., 34

Gonzalez Galvez, G., 245

Gonzalez Gonzalez, J.R., 245

Gonzalez-Rodriguez, M., 223

González, A.H., 122

Gonzalez, C., 333

Gopalakrishnan, S., 185

Goto, S., 149

Gould, S., 321

Goyeau, H., 63

Grad, K., 225

Grady, M., 231

Graham, C., 218

Grassi, B., 275

Grassi, G., 276, 277, 278

Graungaard, T., 61, 67

Gray, L., 257, 355

Greaves, T., 173

Grebenkina, L., 323, 324, 325

Green, E., 294

Greenberg, J., 173 
Gregorc, P., 225

Grinberg, K., 158

Grosembacher, L., 25

Grosman, B., 43, 189

Grøttland, A., 90, 154, 240, 244, 353

Guajardo Jaquez, M., 92

Guastamacchia, E., 278

Guedes, J.A.C., 70

Guedj, S., 164

Gungor, N., 279

Gunnar, H., 244

Guo, Y., 255

Gupta, P., 85

Gupta, V.E., 312

Gutierrez-Ford, C., 131

Gutschek, B., 96

Gyimesi, A., 331

Gyorgy, E., 119

Haahr, H., 68

Haak, T., 314

Habif, S., 273

Hagger, V., 343

Hakaste, L., 133

Hakim, S., 321

Hakim, Y., 141

Haliloglu, B., 280

Halvadjian, I., 201

Hamddan Lachkar, M., 247

Hammond, P., 281

Hána, K., 251

Hannula, P., 282

Hansart, A., 247

Hansen, A.K., 68

Hansen, R., 306

Hardy, T., 66

Harper, A., 159

Harris, S., 309

Hartnell, S., 123, 130

Hartvigsen, G., 154, 240, 248, 339, 354

Harvey, A., 317

Harvey, J., 303

Hasanzad, M., 249

Hashemian, L., 249

Hashimoto, T., 161

Hashimura, K., 161

Hasnani, D., 55

Hasslacher, C., 63, 221

Hatzitolios, A., 95, 272

Haug, C., 54, 297, 298, 359

Hauth, W., 84

Hautier-Suply, O., 160

Havlová, V., 142, 222

Havrlantova, V., 236

Hayashi, T., 149

Hayter, G., 47, 49, 51

Head, R., 314

Heidtmann, B., 101

Heinemann, L., 250, 253

Heise, T., 64, 304

Heller, S., 65, 310

Hellmann, N., 224

Henao Carrillo, D.C., 140, 214, 305

Hendrickx, C., 34
Henn, O., 174

Hepp, D., 238

Herdova, K., 236

Herráiz, L., 212

Herrero Vinas, P., 255

Herrero, P., 127, 163, 186

Heschel, M., 284, 313

Heyman, M., 261

Higashide, T., 161

Hillard, M., 124

Hirano, T., 149

Hiromura, M., 149

Hirose, M., 161

Hjelme, D.R., 105, 350

Hofer, S., 75

Höll, B., 135

Holl, R., 101

Holst, J.J., 72

Holubová, A., 251, 354

Honarvar Nazari, M., 215

Hong, E., 109

Honkasalo, M., 57

Hood, K., 113

Hopkins, D., 232

Hotta, Y., 161

Hovorka, R., 75, 123, 125, 128, 130

Howell, S., 315

Hrachovinova, T., 142

Hradec, J., 142

$\mathrm{Hu}, \mathrm{C} ., 193$

Huang, C., 239

Huang, S., 39, 40, 182

Huddleston, B., 261

Huet, D., 134, 136, 137

Hughes, C., 299

Hummel, M., 96

Hussein, Z., 356

Hyseni, A., 67

Iafusco, D., 301

Iftikhar, M., 232

Iida, M., 176

Ikuta, Y., 176

Imitola Madero, A., 140

Iniesta, J., 333

Iotova, V., 103, 201

Ishii, H., 361

Islas Ortega, L., 92, 245

Itani, M., 264, 289

Iughetti, L., 290

Izzedine, H., 164

Jackson, J., 194

Jafri, R., 28, 124

Jali, S., 103

Jamal, A., 243

James, A., 191

Jana, B., 114

Janez, A., 59

Jangam, S., 47, 51

Jankovic, M.V., 187

Jansa, K., 59

Januszewski, A., 98, 99

Januszewski, A.S., 293
Jaramillo, A., 31

Jarosz-Chobot, P., 274

Järvelä, A., 282

Jedraszko, A., 113

Jenkins, A., 98, 99

Jenkins, A.J., 206, 293

Jennings, P., 365

Jesus, I., 79

Jesus, M.F., 216

Jezek, J., 315

Jia, W., 153, 193, 197

Jiang, B., 234

Jiménez Varas, I., 212

Jimenez, A., 56, 268

Jimenez, J., 94

Jinghua, C., 185

Jirkovska, A., 142

Jirkovska, J., 142, 236

Joakimsen, R., 244

Jochen, S., 267

Johnson, K., 281

Jonak, O., 302

Jones, G., 167, 168

Jones, H., 206

Jones, T., 41, 205

Jones, T.W., 209

Jordan, L., 262

Jordin, A., 185

Jose, R., 115

Joseph, J., 110

Joshee, P., 226

Jothydev, K., 340

Jothydev, S., 80, 115, 340

Jovanovska Mishevska, S., 342

Jugnee, N., 29, 52, 217

Juliussen, R., 284, 313

Justinek, D., 59

Kaczmarzyk, A., 302

Kaiserman, K., 39, 40

Kamecke, U., 54, 297, 298

Kamiuchi, K., 307

Kannard, B., 234

Kapellen, T.M., 75

Karlafti, E., 95, 272

Kartbayeva, G., 348

Karunanithi, M., 355

Kashihara, Y., 161

Kašpar, J., 251

Kataoka, K., 361

Katkó, M., 331

Kato, K., 307

Katzman, P., 87

Kaufman, F., 38, 39, 40, 43, 181, 182, 189

Kaufman, F.R., 211

Kawamura, T., 161

Kawashima, S., 307

Kaya, A., 224, 280, 336

Kazda, C., 66

Kelley, L., 143

Kelly, P., 294, 365, 367

Kempe, H.P., 67

Kerner, W., 101

Kerr, D., 243 
Kesavadev, J., 14, 80, 115, 170, 340

Kessler, K., 152

Kessrwan, C., 116

Khalf, A., 110

Khalifa, A., 264, 289

Khoo Sert Kim, S., 356

Kierulf Åm, M., 111

Kim, C.J., 162

Kim, J.S., 162

Kim, M., 162

Kimura, M., 307

King, B., 60

Klabunde, T., 37

Kleinberg, S., 252

Klemen, D., 165

Klinkenbijl, B., 218

Klonoff, D., 67

Kloppstech, K., 224, 336

Klueh, U., 116

Knuth, M., 221

Kojzar, H., 123, 130

Kolaczynski, J., 303

Kolesnikov, S., 323, 324, 325

Kolesnikova, L., 323, 324, 325

Koleva, R., 201

Kollman, C., 26, 75, 128

Kongable, G., 177, 190

Kongable, G.L., 207

Konoplya, I., 100

Kontoninas, Z., 95, 272

Kordonouri, O., 24, 42, 165

Korek-Abadi, I., 71

Koseoglu, M., 339

Kosta, K., 91

Kothari, M., 13

Kotzakioulafi, E., 272

Kovatchev, B., on behalf of the iDCL Study Group, 106, 108

Kovatchev, B., 6, 10

Kovil, R., 170

Koyama, R., 307

Kožnarová, R., 222

Kreutzer, D.L., 116

Krishnan, G., 80, 115, 340

Krivánek, R., 210, 221

Kriváneková, L., 210, 221

Krompa, K., 134, 136, 137

Kronshage, B., 304

Kruschinski, E., 348

Kulkarni, M., 191

Kulzer, B., 250, 253

Kuo, Y.C., 338

Kurbanyan, L., 85

Kushima, H., 149

Kushner, T., 74

Kuwata, H., 361

Kvapil, M., 295

Laffel, L., 113

Lahtela, J.T., 282

Lalic, N., 19

Lamers, D., 64, 304

Lane, W., 67

Lang, E., 60
Lange, K., 103

Lantigua, H., 299

Lantigua, M.A., 179, 299

Laptev, D.N., 283

Larijani, B., 249

Laryushina, Y., 348

Laszlo, B., 119

Laurenzi, A., 219, 254

Lawton, J., 128

Layne, J., 27, 117

Lázaro-Martín, L., 46

Le Berre, J.P., 134

Lee, J.B., 27, 117

Lee, S., 38, 39, 40, 182, 189

Lee, V., 210

Leelarathna, L., 123, 130

Leite, N., 79

Lema-Perez, L., 337

Lenzi, L., 230

Leohr, J., 66

León Vargas, F.M., 305, 184

Leon-Vargas, F., 118, 144

Leonardo, T., 126

Lepej, D., 42

Levente, K., 119

Levit, S., 71

Levy, B., 231

Lewis, J., 143

Li, K., 255

Li, L., 153

Li, Y., 153

Li, Z., 26

Libiseller, A., 135

Lichtenegger, K., 135

Liebl, A., 65

Lier, A., 151, 152

Lieverse, A.G., 171

Lieverse, C.L.E., 171

Liljenquist, D., 39, 40

Lim, S., 66

Lima, V., 79

Lin, J.T., 85

Lin, T., 199

Lindholm, E., 87

Link, M., 54

Lintereur, L., 43

Liskina, A., 100

Liu, C., 163

Liu, F., 255

Liu, M., 181

Liu, R., 66

Liu, Y.C., 338

Loeum, C., 110

Loh, M.T., 66

Lombardo, F., 230

Löndahl, M., 87

Lonero, A., 230

Longato, E., 133

López Sánchez-Pascuala, J.L., 256

López, B., 172

Lu, W., 153

Lucia, T., 214

Lucisano, J.Y., 85, 206

Lugo, S., 300
Lum, J., 26

Lupiañez, A., 333

Lütolf, S., 258

Luzio, S., 194

Ly, T., 27, 117

Lyngvi Fougner, A., 53, 111

Lyon, J., 78, 271

Maahs, D., 131

Maahs, D.M., 74

Machado, D., 260

Machado, U.F., 70

MacNeil, T., 173

Mader, J., 123

Mader, J.K., 130, 284

Madhu, S., 36

Maffeis, C., 230

Maffioli, P., 58

Maggi, A., 58

Magis Rodriguez, C., 245

Magis-Rodriguez, C., 92

Mahamid, R., 158

Maheno, M., 28, 124

Mahmoodi, A., 145

Maiorino, M.I., 285

Maira, G.J., 214

Makkar, B., 36

Makkar, B.M., 170

Maly, M., 236

Mansoor, I., 319

Mansur, S., 179

Mantovani, L.G., 156

Maran, A., 133

Marchetti, L., 129

Marczak, E., 302

Marescotti, M.C., 203

Margaritidis, C., 95

Maria Alejandra, R., 214

Marigliano, M., 230

Marín Carrillo, L.F., 93

Marin, G., 273

Márquez-Pardo, R., 220

Martha, S.L., 85, 206

Martín-Frías, M., 46

Martinez Ramos Mendez, A., 92, 245

Martínez, C., 333

Martínez, M.J., 333

Martyn-Nemeth, P., 347

Mascarenhas, L., 79

Masel, A., 100

Mašková, J., 222

Massucco, P., 278

Mathieu, C., 48, 61, 310

Mathis, M., 241

Matsumoto, A., 361

Matsumoto, H., 361

Matsumoto, M., 176

Mayer B., Davidson, M.B., 157

Mayer-Davis, E.J., 131

McAuley, S.A., 206

McCue, P., 110

McDonald, L., 112

McGrath, R., 99

McGrath, R.T., 293 
McMahon, C.M., 211

McNamara, C., 343

McVie, R., 279

Mdingi, C., 44, 86

Medvedev, O., 150

Mehta, S., 316

Meiffren, G., 64, 304, 321

Mendoza Romo, M.A., 92

Menefee, E., 279

Meneghini, E., 219

Meneghini, L., 94

Menon, A., 257, 355

Mercier, F., 164

Merino Torres, J.F., 333

Messinger, D., 250, 253

Metso, S., 282

Meyramov, G., 348

Michael, M., 306

Michaud, P., 164

Mihad, H., 314

Mikalsen, M.H., 248

Milena, S., 267

Millán, W., 329

Miller, M., 233

Min, H., 82

Min, J., 183

Ming Yong, C., 262

Mintchev, M., 239

Minuto, N., 230

Miracle Lopez, S., 245

Mirfeizi, M., 249

Mishra, A., 55

Miyahara, Y., 361

Miyazawa, T., 361

Mladenov, V., 201

Mlawa, G., 159, 326

Mohammad Parast, V., 195

Mohammad, M., 356

Mohammedi, K., 164

Mohd Noor, N., 356

Mokáň, M., 291

Molano, A., 118

Molinari, C., 219, 254

Morais, M.R.T., 70

Moramarco, F., 139

Mordvanyuk, N., 172

Moreau, J.P., 319

Moro-oka, Y., 361

Morosanu, A., 146, 147

Morosanu, M., 146, 147

Morris, R., 353

Morros-González, E., 93

Moscardó, V., 50, 148, 186, 213

Moskova, M., 201

Mota, J., 79

Mougiakakou, S., 187, 258, 259

Mouritzen, U., 28

Mozzillo, E., 230

Mueckler, J., 185

Muehlen-Bartmer, I., 308, 309

Mueller, A., 221

Mueller, H., 101

Mujeeb-U-Rahman, M., 215

Müller, A., 210
Muller, I., 24, 42, 165

Muñoz, O., 144

Muñoz, O.M., 140, 246, 305

Munshi, M., 173, 308, 309

Murata, T., 307

Muriel, A., 330

Mužík, J., 251

Mužný, M., 154, 248, 251, 339, 354

N'Guyen, A., 200

Naessens, U., 126

Nagai, Y., 176

Nagaike, H., 149

Nagar, R., 318

Naidis, E., 199

Nakashima, N., 349

Nambam, B., 279

Naranjo, D., 113

Narasaki, K., 307

Nasruddin, A.B., 356

Nategh, R., 341

Navarathna, P., 120

Navodnik-Preloznik, M., 59

Nazarova, A., 102

Neemuchwala, H., 211

Němcová, A., 242

Neves, C., 260, 286

Nevo- Shenker, M., 165

Newswanger, B., 327

Nickerson, J., 252

Nicolucci, A., 218, 267

Nieswandt, A., 24

Nikitina, I., 100

Nikkel, C., 316

Nikolaus, K., 221

Nimri, R., 24, 42, 165

Niño Vargas, R., 92, 245

Nishikawa, N., 161

Nobels, F., 48

Noczyńska, A., 81, 302

Noh, Y., 109

Nørgaard, K., 72

Norlander, L., 82, 183

Northam, L., 128

Novak, M., 317

O'Neal, D.N., 206

O'Sullivan, O., 211

O'Connor, J., 27, 117

O'Donovan, A., 124

Oberlander, N., 320

Ochi, K., 361

Ogawa, Y., 361

Ohara, M., 149

Ohland, S., 179, 299

Oliveira, S., 286

Oliver, N., 29, 50, 52, 148, 163, 213, 262

Onetto, M.T., 275

Osawa, K., 307

Osborn, C.Y., 261

Osmanska, J., 167

Østerskov, A.B., 61

Oukes, T., 107
Paavola, C., 306

Padhye, D.A.

Paknahad, Z., 195

Paldus, B., 206

Panadha, M., 158

Panda, J., 36

Pandis, M., 284

Panteleev, M., 150

Papagianni, M., 91

Parcerisas, A., 175

Pardo, S., 204

Parikh, N., 43, 189

Park, M., 121

Parker, A.S., 56, 268

Parra, R., 260

Parsons, J., 227

Pashagin, A., 222

Pashchenko, I., 150

Pasini, G.F., 58

Passone, C., 165

Patel, F., 55

Pathare, N., 191

Patino-Hernandez, D., 93

Pau, H., 262

Pazos, M., 223

Peeples, M., 265, 266

Pektas, O., 339

Pereira, M., 286

Pérez-Zepeda, M.U., 93

Pertuiset, N., 164

Peterkova, V.A., 283

Petit, J.M., 200

Petrizzo, M., 285

Petrova, C., 201

Petrovski, G., 263, 264, 287, 288, 289, 342, 358

Pettis, R., 317, 320

Pettus, J., 26

Peyser, T., 27, 117

Pfuhl, G., 90

Pfützner, A., 151, 152, 224, 318, 336

Pfützner, A.H., 151, 152, 336

Pfützner, J., 151, 152

Philis-Tsimikas, A., 61

Phillip, M., 24, 42, 165

Pi-ell, T., 180

Piccini, B., 165

Piccinni, B., 230

Piccinno, E., 230

Piche, C., 319

Pickup, J., 78, 271

Pieber R, T., 284

Pieber, T., 68, 123, 135, 315

Pieber, T.R., 65, 130

Pilipenko, V., 102

Pillai, C., 178

Pinhas-Hamiel, O., 39, 40

Pinsker, J., 26

Piona, C., 42, 225

Plank, J., 135

Pleus, S., 54

Plotnikova, O., 102

Plum-Moerschel, L., 66 
Pokramovich, U., 269, 270

Poláček, M., 251

Polanco Preza, M.A., 92

Polsky, S., 226

Polyanskaya, A., 100

Popescu, L., 69

Pories, W., 22

Pors, A., 238

Potier, L., 164

Pöttler, T., 284

Pouwer, F., 34

Prazny, M., 142

Predieri, B., 290

Prestrelski, S., 327

Price, D., 60

Pridavkova, D., 291

Pritlove, C., 227

Provenzano, F., 169

Provenzano, V., 169

Pustovalova, O., 354

Pyle, L., 226

Pyšná, A., 242

Qiao, Y., 116

Quinn, L., 121

Quirós, C., 46, 88, 202

Rabbone, I., 165, 230

Rachel, B., 165

Raddino, R., 58

Raeiszadeh, M., 319

Raisoni, B., 228, 368

Rajesh, R., 338

Rama Chandran, S., 328

Ramachandran, L., 80, 115, 340

Rami-Merhar, B., 75

Ramirez-Rincón, A., 337, 344

Ramljak, S., 224

Ranamukha, S., 319

Randell, T., 128

Rangel Gerrero, H.G., 92

Ranjan, A., 72

Ranson, A., 64, 304, 321

Rapini, N., 230

Raposo, F., 103

Raskin, P., 65

Rastogi, R., 86

rathi, N., 363

Ray, S., 281

Raz, I., 180

Reali, F., 129

Rebolledo, M., 140, 214

Reddy, M., 29, 50, 52, 148, 213

Reddy, S., 66

Redondo, C., 286

Reeh, W., 318

Regincos, J., 175

Reidy, C., 292

Reiterer, F., 89, 166, 229

Remus, K., 24

Renard, E., 17, 67

Rezaninová, L., 242

Reznik, Y., 16

Rhinehart, A., 77
Ribu, L., 90

Riddell, M., 131

Riddle, S., 177

Riddle, S.A., 207

Rihankova, R., 142

Riley, K., 317

Rini, C., 320

Rios, C., 329, 330

Ritzel, R., 62, 308, 309

Rivadeneira, P.S., 122, 188, 344

River-Davila, M., 178

Rivera - Davila, M., 300

Rivera-Davila, M., 299

Rivera, M., 179

Roberts, A., 205

Roberts, B., 320

Robledo, M.A., 140

Rodrigo Cano, S., 333

Rodriguez Sanchez, E., 245

Rollato, A.S., 301

Rom, V., 180

Romano, O., 285

Romero Zazueta, A., 245

Rondón, M., 214, 305, 140

Rosales, N., 184

Rose, D., 318

Rose, L., 61

Rosenstock, J., 62

Rossetti, P., 186

Rossi, M.G., 254

Roussel, R., 62, 309

Roy, A., 43, 189

Rozas, P., 216

Roze, S., 57

Roze, S.R., 128

Rozner, A., 199

Ruan, Y., 75, 123, 130

Rubio, C.P., 305

Rubio, N., 178, 299, 300

Runkle de la Vega, I., 212

Russell-Jones, D., 61, 69, 310

Russell, A., 257, 355

Russell, S., 28, 124, 131

Russo, E., 351

Ruszala, A., 165

Rutigliano, I., 165

Saad, M., 32

Saadtjian, L., 164

Saber, S., 159

Saboo, B., 15, 36, 55, 80, 170, 340

Sach-Friedl, S., 68

Sainsbury, C., 167, 168

Sainz de la Maza Viadero, M.E., 92, 245

Saiyed, M., 55

Sakane, N., 307

Sakka, D.S., 165

Saleem, M., 326

Salem, H., 32

Sally, R., 367

Sanchez Peña, R., 25

Sanchez, P., 208

Sandro, G., 267
Sankaranarayanan, S., 74

Santi, L., 129

Sarnataro, A., 285

Sarno, G., 346, 351

Sauque Reyna, L., 245

Saura, G., 169

Sawaki, H., 307

Sayinbatur, B., 280

Scaramuzza, A., 230

Scavini, M., 156, 219, 254

Scheerer, M., 24

Schiaffini, R., 230

Schiavon, M., 35, 37

Schierloh, U., 76, 125

Schmidt, S., 72

Schnell, O., 96

Schøndorff, P.K., 313, 284

Schramm, W., 250, 253

Schrangl, P., 89, 229

Schultes, B., 69

Schwarzenbacher, D., 284

Schwingenschuh, S., 313

Scibona, P., 25

Scorsone, A., 169

Scott, E., 99

Scott, E.M., 128

Scott, E.S., 293

Scuntero, P., 277

Sears, L.E., 261

Sebagh, N., 164

Segal, E., 8

Segall, T., 165

Seifert, M., 81, 302

Selagamsetty, R., 124

Selb, J., 30

Semenova, N., 325

Sencan, M., 215

Sentsova, T., 102

Sereno, J.E., 188

Servine, M., 112

Setford, S., 231

Shah, A., 36, 170, 178

Shah, S., 55

Shaikh, F., 13

Shaikh, M., 36, 170

Shankar, A., 80, 115, 340

Shapley, J., 160

Sharafetdinov, K., 102

Sharifiheydarabad, H., 341

Sharma, A., 243

Sharma, H., 191

Sharma, R.K., 312

Shaybek, A., 348

Sheng, C., 153

Shestakova, T., 150

Shi, L., 233

Shin, J., 38, 39, 40, 181, 182

Shin, L., 181

Shirakawa, I., 361

Shojaeizadeh, E., 196

Shomali, M., 265, 266

Shulman, D., 39, 40

Sieber, J., 37, 62, 177

Siesky, A., 306 
Sifontes-Dubon, M.A., 223

Simmons, D., 204

Simonovich, V., 25

Simunovic, M., 165

Sinner, F., 313

Skibová, J., 142, 242

Slattery, D., 232

Slegtenhorst, S., 75, 128

Slyne, C., 173

Smith, G., 41, 205, 209

Smith, M., 294

Snel, T., 171

Snell-Bergeon, J., 226

Snyder, J., 303

Solar, S., 236

Somaiah, S., 235

Sosale, A., 36, 170, 235

Sosale, B., 235

Soula, O., 64, 126, 304, 321

Soula, R., 126, 321

Spano, L., 169

Sparacino, G., 45, 133, 155, 237

Spatz, J., 152

Speight, J., 34

Sperling, M., 7

Sperry, A., 306

St Clair, R., 319

Staal, O.M., 53

Stanga, Z., 259

Stathopoulou, T., 258

Stavdahl, Ø., 53, 105, 350

Stechova, K., 295

Steele, C., 165

Stefankova, J., 142

Stein, R., 165

Steineck, I., 72

Stępkowski, M., 302

Stergiou, I., 95

Sternad, S., 357

Stettler, C., 187

Stipancic, G., 103

Stoeber, B., 319

Stoeva, I., 201

Stolletz, N., 135

Stone, M., 38

Strange, P., 233

Strasma, P., 110

Strauss, K., 294

Strobl, S., 151, 152

Strojek, K., 69

Sturm, A., 30

Suganami, T., 361

Sullivan, A., 234

Šumník, Z., 103

Sun, Q., 187

Šuput Omladič, J., 165

Susha Nair, A., 235

Šutarík, L;., 291

Sveshnikova, A., 150

Swanson, V., 26

Szypowska, A., 103

Tabatabai, I., 82, 183

Tabor, A., 274
Takeishi, S., 311

Takekoshi, S., 311

Tanabe, Y., 149

Tanaka, M., 361

Tannen, A.J., 50

Tanzanakis, A., 127

Tapia, J., 333

Tassone, F., 278

Taub, M.B., 207

Tauschmann, M., 75, 128

Tavera Hernandez, M., 92

Taybani, Z., 331

Taylor, D., 343

Taylor, S., 243

Tenés, S., 333

Teng, S., 321

Terjung, N., 273

Thabit, H., 123, 130

Thangappan, A., 235

Thomsen, K.M.D., 68

Thorpe, A., 128

Thuan, J.F., 136, 137

Tigishvili, L., 345

Tinti, D., 165, 230

Tkachenko, P., 89, 229

Todorova-Jovanovska, B., 287

Toffoli, C., 290

Tomaskova, G., 236

Tomic, S., 104

Tomoyasu, M., 149

Tone, A., 307

Tonolo, G., 278

Torjman, M., 110

Torrent-Fontbona, F., 172

Torres-Barea, I., 220

Toschi, E., 173, 174

Toyoda, M., 307

Travert, F., 134, 136, 137

Treiber, G., 284

Trevelyan, N., 128

Triberti, S., 254

Tritto, I., 58

Trombetta, M., 129

Troub, T., 182

Tsanakas, I., 91

Tsikarishvili, N., 345

Tsiroukidou, K., 91

Tsotoulidis, S., 95

Tsuboi, H., 311

Tsui, V., 210

Tsuruo, M., 307

Tubili, C., 278

Tuhackova, M., 295

Tuomi, T., 133

Tzamouranis, K., 101

Tziomalos, K., 95

Uehlinger, D., 258

Ulbrich, S., 54, 359

Umbach Hansen, H.M., 53

Umer, F., 264, 289

Umpierrez, G., 140

Undurti, D., 73, 332

Urbaniec, K., 295
Væver Bysted, B., 28

Vaillant, G., 200

Vaillant, M.T., 76, 125

Valenzano, M., 277

Valenzuela Montoya, J.C., 92

Valenzuela-Lara, M., 92, 245

Vallejo, M., 344

Vamvakis, A., 91

van Bon, A.C., 107

van Vroenhoven, J.C.M.D., 171

Van Wyk, E., 224

Vanbesien, J., 247

Vanis, M., 295

Vanslyke, S., 45

Vasiloglou, M.F., 259

Vazeou, A., 103

Vecchio, M., 316

Veeze, H., 103

Vehí, J., 175, 202

Velasco, M., 329, 330

Venerova, J., 236

Ventrici, C., 230

Vergès, B., 200

Vermeulen, B., 247

Vespasiani, G., 63, 267

Vesper, I., 250, 253

Vettoretti, M., 45, 155, 237

Vidrio Velazquez, M., 92, 245

Vigersky, R., 234

Vigier-Simmore, N., 134, 137

Villa, R., 346, 351

Viñals, C., 88

Visentin, R., 35, 37

Vlasáková, M., 251

von Raesfeld, A.M., 107

Vorgucin, I., 104

Wadhwani, D.A., 114

Wadwa, R.P., 27, 117

Wahlqvist, P., 296

Waldenmaier, D., 297, 298, 359

Walker, T., 56

Wang, G., 239

Wang, J., 197

Wang, X., 62

Wang, Y., 153

Warner, J., 296

Watanabe, H., 176

Watanabe, T., 307

Weber, A., 238

Wehbe, M., 319

Weinheimer, M., 177, 190

Weinheimer, M.H., 207

Weissberg-Benchell, J., 113

Weissmann, J., 84, 250, 253

Welsh, J., 56

Welsh, J.B., 268

Westerbacka, J., 312

Wienbarg, I., 84

Wiesinger, T., 135

Wilinska, M., 123

Wilinska, M.E., 75, 125, 128, 130

Wilkes, R., 239

Woloschak, M., 63 
Woo, V., 310

Wood, M., 39, 40

Wosková, V., 242

Wu, D., 43, 189

Wu, H., 306

Xu, D., 98

Xu, Y., 33, 47, 49, 51

Yadav, V.K., 363

Yadid-Pecht, O., 239

Yafi, M., 178, 179, 299, 300

Yamada, H., 361

Yamamoto, R., 349
Yamamoto, T., 149

Yepez Rodriguez, A.E., 92

Yeşiltepe Mutlu, G., 42, 225

Yochpaz, S., 180

Yoshida, M., 349

Yuan Ling Lim, A., 328

Zaghlol, A., 32

Zaharieva, D., 131

Zakrzewska, K., 96

Zanfardino, A., 301

Zapata-Madrigal, G., 344

Zarra, E., 278

Zhang, N., 308, 309
Zhang, S., 153

Zhao, C., 83, 132

Zhao, H., 132

Zheng, M., 252

Zhong, A., 211

Zhou, F.L., 94, 312

Zhou, J., 153

Zhuplatov, S., 204

Ziegler, R., 297, 298

Zivkovic, M., 263, 287, 288, 342

Zivony, A., 71

Zorn, T.M.T., 70

Zubkiewicz-Kucharska, A., 81, 302 